\title{
La Biodiversidad y su transposición didáctica en el currículo de Primaria en las Leyes Orgánicas de Educación desde la LOGSE (1990)
}

\author{
Jose Manuel Escobero Rodríguez \\ Colegio Público Andrés Segovia. Tutor de Primaria. Granada. España \\ jmescobero@gmail.com \\ ORCID: https://orcid.org/0000-0002-2939-4555
}

[Recibido: 24 Febrero 2020. Revisado: 14 Abril 2020. Aceptado: 28 Abril 2020]

\begin{abstract}
Resumen: Existen muy pocos estudios sobre el tratamiento de la biodiversidad en el currículo de Primaria, así como sobre la transposición didáctica desde el Saber Sabio de este concepto a las Leyes Orgánicas de Educación. Este estudio intenta profundizar en la calidad de ese primer paso transpositivo en el currículo de Primaria, a través de un análisis cualitativo y cuantitativo de dicho proceso mediante una metodología que cuantifica y evalúa el tratamiento de la biodiversidad a nivel nacional y autonómico andaluz. Se comprueba que la transposición de la biodiversidad es incompleta, desigual y desestructurada. Se analizan los principales errores y carencias del proceso, y se señalan posibles actualizaciones, comparando ambos niveles a través de las distintas Leyes hasta la actual LOMCE. La conclusión final apunta a que no se facilita un aprendizaje de calidad con el actual marco curricular.
\end{abstract}

Palabras clave: biodiversidad; transposición didáctica; currículo de primaria; ley educativa.

Biodiversity and its Teaching Transposition in the Elementary Curriculum in the Organic Education Laws since the LOGSE (1990)

Abstract: There are few studies on the treatment of biodiversity in the Primary Education curriculum. There are even less on the didactic transposition of the Scientific Knowledge behind this concept to the Laws on Education. This study tries to delve into the quality of that step by offering both with a qualitative and quantitative analysis on biodiversity's transposition; referred to both the national level and Andalusia one. Major mistakes and shortcomings of the transposition are analyzed, and possible updates are pointed out while comparing national and regional scopes. Also, we assess the chronological evolution of the process through various laws up the current LOMCE; we found biodiversity's transposition is incomplete, unbalanced and unstructured, with differences between the national and regional level; finally, no significant learning on biodiversity seems to be guaranteed .

Keywords: biodiversity; didactic transposition; elementary curriculum; educational law .

Para citar este artículo: Escobero, J. M. (2020) La transposición didáctica de la Biodiversidad y su tratamiento en el currículo de Primaria en las Leyes Orgánicas de Educación desde la LOGSE (1990). Revista de Educación Ambiental y Sostenibilidad 2(1), 1303. doi: 10.25267/ Rev_educ_ambient_sostenibilidad.2020.v2.i1.1303

\section{Introducción}

Biodiversidad es un término reciente, complejo, y muy difundido (Tellería, 2002) desde su primer uso en 1986 (Wilson, 1986; 1988). Se asoció con diversidad de formas vivas, con los factores relacionados con su pérdida y con aquellos que la incrementan o mantienen, involucrando diversas ramas científicas (Wilson en Núñez, González-Gaudiano y Barahona, 2003). Desde los 90, este término, junto con 
conservación y ecosistema han pasado a ser de dominio público, aunque a veces con dudoso rigor científico.

La biodiversidad se ha definido como una propiedad específica de un área (DeLong, 1996), estructurada en elementos y niveles (Gaston y Spicer, 1998), que señala las relaciones entre componentes bióticos y abióticos del sistema (Swingland, 2001), y donde el ser humano adquiere cada vez mayor preponderancia (Ellis y Ramankutty, 2008; Tellería, 2012). En la Conferencia de las Naciones Unidas sobre Ambiente y Desarrollo, la ONU añade al concepto de biodiversidad "valores ecológicos, genéticos, sociales, económicos, científicos, educativos, culturales, recreativos y estéticos, así como la evidencia de la necesidad de mantenerla para la evolución y para el mantenimiento de los sistemas necesarios para la vida de la biosfera" (ONU, 1992), abriendo el camino para la definición de desarrollo sostenible y educación para la sostenibilidad (DETR, 1999). El PNUMA (Programa de las Naciones Unidas para el Medio Ambiente) considera su pérdida "como una de las mayores amenazas para nuestro mundo", afirmando que "durante los tres últimos decenios, la disminución y la extinción de especies se han afirmado como problemas ambientales de la mayor importancia" (PNUMA, 2002). Las presiones social y científica han venido incrementando la toma de acuerdos y acciones para su preservación. La educación para la sostenibilidad implica procesos participativos, críticos, sociales y orientados a la acción local (Peña, Gil y Cañal, 2010). Autores como Van Weelie (2002) comprobaron que su dialéctica puede ser un buen instrumento didáctico, y que el conflicto de intereses facilita una postura crítica en las aulas (Gómez y Bernat 2010), considerando una solución a escala global, porque los problemas lo son (Peña et al., 2010). La formación necesaria para su desarrollo, no obstante, es escasa en los futuros docentes (Calero, Mayoral, Ull y Vilches, 2019), y se apunta al hecho de utilizar la inclusión de esta idea como competencia, mediante un proceso de investigación (Estrada y González, 2004) e impregnación en la cultura de la sostenibilidad (Martín-Vivaldi, Gutiérrez y Arjona, 2000).

Este perfil cognitivo de la biodiversidad dificulta su comunicación y enseñanza (Núñez et al., 2003), y convierte en fascinante su adaptación educativa, especialmente en la Educación Primaria (Gómez y Bernat, 2010), lo que genera problemas en varios niveles: a) reducirla a intervenciones conservacionistas; b) su multidisciplinariedad en un currículo no integrado dificulta su aprendizaje (Gayford, 2000) y genera lagunas en docentes que muestran esas lagunas y las transmiten (Sánchez-Cañete y Pedrajas, 2010); c) ausencia de una definición de consenso (Dangerfield y Pik, 1999); y d) percepción sobre la falta de una política oficial e interés en su tratamiento en las aulas (Lindemann-Matthies et al., 2009).

La estructura gnoseológica del contenido curricular afecta a su tratamiento educativo (Gascón, 1993). Según la Teoría de la Transposición Didáctica (Bosch y Gascón, 2009) resulta fundamental contemplar la adecuación de contenidos complejos, y que a su carácter exógeno respecto a la escuela añaden su propia discusión epistemológica. La TTD (Chevallard,1985) y la Teoría Antropológica de lo Didáctico (Chevallard, 1992a, 1992b, 2002; Bosch y Gascón, 2006; Gascón, 2011) proporcionan las herramientas con las que pretendemos analizar cómo se trata el concepto de la biodiversidad en el sistema educativo en el ámbito institucional, o primer nivel transpositivo, según la TTD. 
El trabajo previo en este campo ha referido en su mayoría la transposición de la biodiversidad en los libros de texto (Fuentes, Barros y Losada, 2008; 2010). En Primaria la transposición es inadecuada e irrelevante (García, 2013), reducida a su nivel conceptual (Caurin y Martinez Penella, 2013). En Secundaria la transposición autonómica es mejor valorada (Fuentes et al., 2008), aunque la biodiversidad suele reducirse a la riqueza biológica (Bermúdez, De Longhi, Díaz, y Catalán, 2014), indicando no solo inexactitud científica, sino ausencia de actualización (España y Prieto, 2010). Trabajos relevantes en el ámbito de la Educación ambiental, que hoy han desembocado en la Educación para la Sostenibilidad, no han hecho la mella necesaria en los programas de formación de los futuros profesores y profesoras (Herrería, y Caveda, 2010; Calero et al. 2019). Este estudio es escaso en la formación de maestros y maestras.

Rastrearemos la presencia del metaconcepto biodiversidad (Armúa de Reyes, Guirardo y Brataszczuk, 2005; Vázquez-Ben y Bugallo-Rodríguez, 2018) en las leyes educativas desde 1990 hasta la actualidad (LOGSE, LOE y LOMCE) en la Etapa de Primaria, puesto que indica su relevancia social. Se comparará el nivel ministerial con el nivel autonómico andaluz. Para ello realizaremos un análisis cualitativo y cuantitativo de su presencia en dichas leyes, la rigurosidad con que es presentada, cómo se plantean y articula los distintos niveles que la conforman. Nuestra hipótesis es que el tratamiento de la biodiversidad en el primer escalón transpositivo es pobre, y que esta no se representa suficientemente en nuestras leyes educativas.

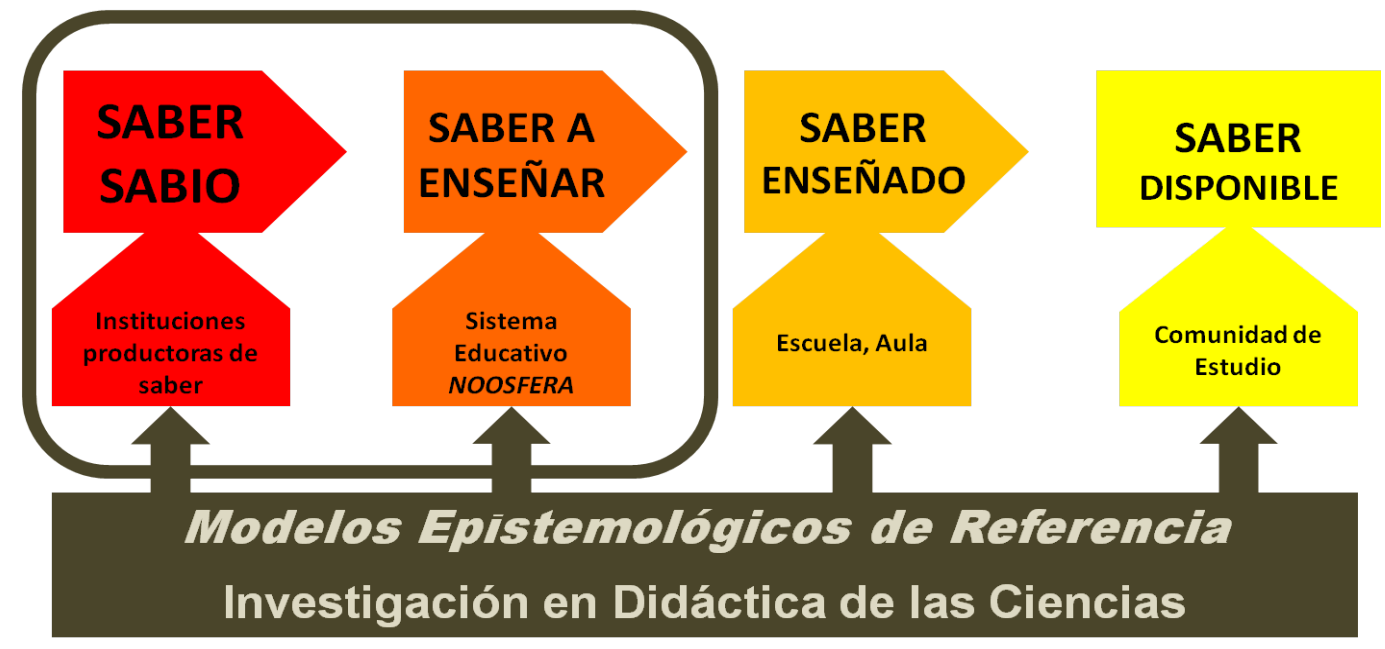

Figura 1. La posición del investigador en la TAD. Adaptado de Bosch y Gascón (2009)

En la propuesta normativa e institucional (Saber a Enseñar, Figura 1) mencionaremos algunas sugerencias metodológicas que sirvan de orientación a aquellos docentes de Primaria que deseen abordar esta temática en sus aulas, y sin cuyo aprovechamiento este trabajo no tendría sentido.

\section{Método}

En línea con Vázquez-Ben y Bugallo-Rodríguez (2018), no pretendemos el desarrollo curricular de la biodiversidad como un simple contenido, sino que, debido a su importancia y a la inclusión de la misma idea de la biodiversidad en la categoría de big idea, ideas nucleares o metaconcepto, debe incluirse un constructo progresivo de modelos científicos que posibilite el éxito de la compresión del concepto desde la 
etapa de Infantil. En este sentido, el instrumento de evaluación utilizado (árbol de codificación) supone una propuesta que también puede ayudar a tratar la progresión de las ideas implicadas en la comprensión de la biodiversidad, en las actitudes a desarrollar para con ella (sin las cuales su conocimiento resulta vano), y en la idoneidad de los modelos utilizados en este proceso.

Nuestro análisis de la biodiversidad considera los componentes: genético, especies/poblaciones, y comunidades/ecosistemas (Gaston y Spicer, 1998) con sus atributos de composición, estructura y función (Redford y Richter, 1999). Finalmente definimos biodiversidad en una doble dimensión atendiendo a biodiversidad estructural y biodiversidad funcional (Tellería, 2012), con un tercer nivel a efectos de inventario, que sería el composicional. Este tercer nivel lo situamos dentro de la biodiversidad estructural, señalando la diversidad de la vida individual y colectivamente considerada, junto a los distintos niveles de organización (los seres vivos clasificados jerárquicamente en grupos taxonómicos mutuamente excluyentes), junto a las especies, genes, poblaciones y ecosistemas. Para la biodiversidad funcional reconocemos los procesos ecológicos y evolutivos que garantizan la supervivencia de los seres vivos (Noss y Cooperrider, 1994).

Se han analizado todos los textos de la Ley Orgánica de Ordenación General del Sistema Educativo de 1990, la Ley Orgánica de Educación de 2006 y la actual Ley Orgánica para la Mejora de la Calidad Educativa de 2013, según el Boletín Oficial del Estado (BOE) y el Boletín Oficial de la Junta de Andalucía (BOJA). Para incluir una cita los textos debían referirse a:

- Objetivos, fines, finalidades.

- Contenidos, en cualquiera de sus acepciones, siendo dichos contenidos aplicados de una manera muy diferente de una Ley de Educación a otra.

- La Evaluación no ha sido incluida, al considerar que hace referencia a objetivos y contenidos desarrollados con anterioridad, pudiendo generar redundancia. Exceptuamos los estándares de evaluación de la LOMCE, que se sitúan más cerca de estándares curriculares que evaluativos, porque derivan de procedimientos cercanos al mundo laboral y no al educativo (Valverde, 2009).

Solo se escogió para el análisis una cita de cada tipo y Ley: aquella que más puntuación obtuviese. Si el texto en cuestión aparecía repetido de igual o muy parecida forma en distintos ciclos, solo se escogía el de más complejidad. Este análisis se efectuó con dos herramientas definidas a priori (y sancionadas por un comité de expertos):

- Una tabla de presencia de los niveles de la biodiversidad.

- Una tabla de valoración que califica dichos niveles (criterios) respecto a un patrón de complejidad creciente, que a falta de otra denominación (Schnurr, De Santo, Green y Taylor, 2015; Blackmore, y Doole, 2013), llamamos árbol de codificación (Tabla 1). 
Tabla 1. Árbol de codificación

\begin{tabular}{|c|c|c|c|c|c|c|c|c|c|c|c|}
\hline \multirow[t]{2}{*}{ valeer } & \multirow{2}{*}{$\begin{array}{l}\text { 1. NIVEL } \\
\text { COMPOSICIONAL }\end{array}$} & \multicolumn{6}{|c|}{ 2. NIVEL ESTRUCTURAL } & \multicolumn{4}{|c|}{ 3. NIVEL FUNCIONAL } \\
\hline & & $\begin{array}{l}\text { 2. 1. Distintos Reinos } \\
\text { y Dominios } \\
\text { clasificatorios del } \\
\text { mundo vivo }\end{array}$ & $\begin{array}{l}\text { 2.2. Nivel } \\
\text { Especie }\end{array}$ & $\begin{array}{l}\text { 2.3. Nivel } \\
\text { Genético }\end{array}$ & $\begin{array}{l}\text { 2.4. Nivel } \\
\text { Población }\end{array}$ & \multicolumn{2}{|c|}{ 2. 5. Nivel Ecasistema } & $\begin{array}{l}\text { 3. 1. El Hombre } \\
\text { como agente } \\
\text { respecto a la } \\
\text { Baodiversidad }\end{array}$ & $\begin{array}{l}\text { 3.2. Procesos } \\
\text { Ecológicas }\end{array}$ & \multicolumn{2}{|c|}{ 3.3. Procesos Evolutivos } \\
\hline 1 & $\begin{array}{l}\text { 1. 1. Aparece el } \\
\text { grupo de palabras } \\
\text { diversidad de seres } \\
\text { vinos. }\end{array}$ & $\begin{array}{l}\text { 2.1.1. Diferencia entre } \\
\text { animales } y \text { plantas }\end{array}$ & $\begin{array}{l}2.21 \\
\text { Menciona } \\
\text { el término } \\
\text { especie }\end{array}$ & $\begin{array}{l}2.3 .1 \\
\text { Menciona la } \\
\text { diferencia } \\
\text { fenotípica de } \\
\text { manera simple } \\
\text { y puntual }\end{array}$ & $\begin{array}{l}2.4 .1 \\
\text { Menciona las } \\
\text { poblaciones } \\
\text { constituidas } \\
\text { por los } \\
\text { individuos de } \\
\text { una misma } \\
\text { especie }\end{array}$ & $\begin{array}{l}\text { 2. 5.1. Utiliza el } \\
\text { paisaje, haciends } \\
\text { diferentes tipos } \\
\text { componentes }\end{array}$ & $\begin{array}{l}\text { Incepto de } \\
\text { alusión a sus } \\
\text { e }\end{array}$ & $\begin{array}{l}\text { 3.1.1. Mención del } \\
\text { hombre o sus } \\
\text { actividades como } \\
\text { agente } \\
\text { fundamental en el } \\
\text { funcionamiento de } \\
\text { los ecosistemas y } \\
\text { comunidades }\end{array}$ & $\begin{array}{l}3.2 .1 \\
\text { Menciona } \\
\text { algún proceso } \\
\text { ecológico }\end{array}$ & $\begin{array}{l}\text { 3.3.1. Menciona } \\
\text { las funciones } \\
\text { vitales como } \\
\text { características } \\
\text { de los seres vivos }\end{array}$ & \\
\hline$\overline{2}$ & $\begin{array}{l}\text { 1.2. Aparece el } \\
\text { concepto } \\
\text { Buodiversidad }\end{array}$ & $\begin{array}{l}\text { 2.1.2. Diferencia entre } \\
\text { animales, plantas y } \\
\text { microorganismos } \\
\text { pertenecientes a otras } \\
\text { categorías } \\
\text { taxonómicas }\end{array}$ & & $\begin{array}{l}\text { 2. } 3.2 \text {. } \\
\text { Menciona la } \\
\text { riqueza } \\
\text { genética como } \\
\text { diferencia } \\
\text { entre los } \\
\text { individuos de } \\
\text { una especie }\end{array}$ & & $\begin{array}{l}\text { 2. } 5.2 \text {. } \\
\text { Menciona el } \\
\text { concepto de } \\
\text { ecosistema, sin } \\
\text { hacer alusión a } \\
\text { sus diferentes } \\
\text { componentes }\end{array}$ & & $\begin{array}{l}\text { 3.1.2. Se describen } \\
\text { o mencionan } \\
\text { relaciones de } \\
\text { responsabilidad } \\
\text { sobre esa } \\
\text { incidencia }\end{array}$ & $\begin{array}{l}3.2 .2 \text {. } \\
\text { Diferencia } \\
\text { entre algunos } \\
\text { tipos de } \\
\text { procesos } \\
\text { ecológicos } \\
\text { (fotosintesis, } \\
\text { ciclos de } \\
\text { nutrientes, } \\
\text { etc) }\end{array}$ & $\begin{array}{l}\text { 3.3.2. Idem como } \\
\text { características } \\
\text { diferenciadoras y } \\
\text { que confieren } \\
\text { biodiversidad }\end{array}$ & $\begin{array}{l}\text { 3.3.3. Menciona la } \\
\text { Evolución como } \\
\text { característica de } \\
\text { los seres vivos }\end{array}$ \\
\hline 3 & $\begin{array}{r}\text { 1.3. Relaciona al } \\
\text { menos un primer } \\
\text { nivel de } \\
\text { Biodiversidad con la } \\
\text { diversidad de } \\
\text { tawones de seres } \\
\text { vivos }\end{array}$ & $\begin{array}{l}\text { 2.1.3. Diferencia entre } \\
\text { los distintos tipos de } \\
\text { seres vivos, realizando } \\
\text { mención expresa de } \\
\text { animales, plantas, } \\
\text { microorganismos (en } \\
\text { el sentido antes } \\
\text { descrito) y alguna- } \\
\text { todas otra categoria } \\
\text { taxonómica } \\
\text { equivalente }\end{array}$ & & $\begin{array}{l}2.3 .3 . \\
\text { Menciona la } \\
\text { riqueza } \\
\text { genética como } \\
\text { diferencias } \\
\text { entre los } \\
\text { genes/alelos de } \\
\text { un conjunto de } \\
\text { individuos de } \\
\text { una especie }\end{array}$ & & $\begin{array}{l}\text { 2.5.3. } \\
\text { Menciona el } \\
\text { concepto de } \\
\text { ecosistema, } \\
\text { haciendo } \\
\text { alusión a sus } \\
\text { diferentes } \\
\text { componentes }\end{array}$ & $\begin{array}{l}\text { 2. } 5.4 \\
\text { Menciona } \\
\text { distintos } \\
\text { tipos de } \\
\text { ecosistema }\end{array}$ & $\begin{array}{l}\text { 3.1.3. Se solicita el } \\
\text { desarrollo de } \\
\text { actitudes } \\
\text { responsables } \\
\text { sobre esa } \\
\text { incidencia de } \\
\text { mejora, gestión, } \\
\text { conservación o } \\
\text { recuperación }\end{array}$ & & & $\begin{array}{l}\text { 3.3.4. Menciona la } \\
\text { Extinción de } \\
\text { especies como } \\
\text { proceso evolutivo }\end{array}$ \\
\hline 4 & & & & & & & & $\begin{array}{l}\text { 3.1.4. Se aportan o } \\
\text { proponen } \\
\text { intervenciones } \\
\text { concretas en dicha } \\
\text { linea directa o } \\
\text { indirectamente }\end{array}$ & & & $\begin{array}{l}\text { 3.3.5. Menciona la } \\
\text { adaptación como } \\
\text { proceso evolutivo }\end{array}$ \\
\hline 5 & & & & & & & & & & & $\begin{array}{l}\text { 3.3.6. Menciona } \\
\text { otros procesos } \\
\text { evolutivos (ciclos } \\
\text { vitales, } \\
\text { dispersión, } \\
\text { relaciones } \\
\text { interestecificas, } \\
\text { seevolucion, etc.) }\end{array}$ \\
\hline
\end{tabular}


Un texto puede tener varias puntuaciones, aunque jamás se puntuó más de una vez atendiendo a criterios de la misma fila. De tal manera aseguramos la presencia de los distintos niveles y atributos descritos por Noss (1990). Al finalizar el proceso se contaba con 160 citas valorables.

Los textos se procesaron individualmente, con un entrenamiento inicial y una comprobación final (Saldaña, 2009; Fernández-Río y Suarez, 2014), sustituyendo así la evaluación interpares, siguiendo el método de comparación constante (Lincoln y Guba, 1985; Libarkin y Kurdziel, 2002; Jackson, Drummond y Camara, 2007). Es decir, la búsqueda se realizó varias veces y se escogieron los textos que figuraban constantemente, una vez las repetidas observaciones fueron consistentes en el tiempo. Aunque se han seguido las pautas generales de estos estudios (Makarova y Birman, 2015; Janghorbank, Roudsari, Taghipour y Abbasi, 2014; Fernández-Río y Suárez, 2014), no se localizó en la bibliografía un trabajo similar al presente en cuanto a la transposición de la biodiversidad, conteniendo: análisis cualitativo de un concepto altamente complejo, valoración jerárquica del mismo, y usos de instrumentos diseñados a priori y chequeados mediante el control de un comité de expertos.

\section{Comité de Expertos}

El Comité de Expertos lo constituyó un grupo de especialistas en distintas ramas de la Biología, junto a sabios en el campo de la Didáctica, que consensuaron las formas finales de las herramientas de trabajo (Fernández-Río y Suárez, 2014). Su punto de vista ha dado profundidad y amplitud de miras a esta valoración.

\section{Taxonomía de lo vivo}

La valoración de este nivel ha entrañado cierta dificultad, algo de esperar en un tema biológicamente tan controvertido. Como docente, justifico trabajar con una unidad mínima de biodiversidad, que sugiero sea el ser vivo, de forma que se puede establecer su definición en virtud de sus funciones vitales (nutrición, relación y reproducción) y no en la falsa serie "nacer, crecer, reproducir, morir". Un adecuado aprendizaje de lo que es un ser vivo evitaría los problemas sobre la especie (JiménezTejada, Sánchez, Romero y González, 2014) y la población (Jiménez-Tejada, SánchezMonsalve y González-García, 2013).

\section{Procesos ecológicos}

En el primero de los Congresos Mundiales sobre Medio Ambiente (celebrado en Estocolmo en 1972) ya se realiza un llamamiento a un desarrollo responsable y a la visión multidisciplinar de la Educación Medioambiental (Eschenhagen 2007; Herráiz y del Valle, 1995). Esta perspectiva influye transversalmente en todos los posicionamientos de los encuentros posteriores y en los programas de intervención del PNUMA (2002). La Conferencia de Río (celebrada en 1992) alude a la "educación como una estrategia fundamental para la conservación", y puede hablarse de la Educación Ambiental en el marco curricular de las Ciencias con un carácter metadisciplinar (Gómez y Bernat 2010). Hemos querido añadir ésta a las otras dimensiones de la biodiversidad. 


\section{Procesos evolutivos}

La dificultad de la valoración consistió en su jerarquización. Pendiente de una mayor profundización se graduaron en virtud de la dificultad creciente en reconocerlos. La adaptación fue una de las imprescindibles aportaciones del comité en este punto.

\section{El Ser Humano como agente}

Es reconocida la capacidad de la especie humana para modificar la biosfera, y hoy existe un amplio consenso en considerar que los grandes problemas que atañen a nuestro planeta en general, y especialmente a la biodiversidad, tienen un origen antrópico. Aquí recogemos las diferentes posibilidades de intervención humana, primando las opciones de acción.

\section{Resultados}

Los resultados aparecen resumidos en las tablas 2 y 3 y representados en los gráficos de las figuras 2 y 3. 
Tabla 2. Matriz del número de textos con la valoración obtenida de cada uno.

\begin{tabular}{|c|c|c|c|c|c|c|c|c|c|c|c|c|c|c|c|c|c|c|}
\hline$\sum_{\text {L }}^{\text {尼 }}$ & 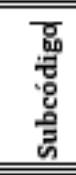 & 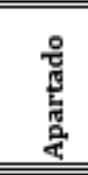 & 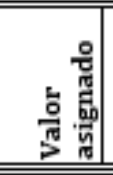 & 崖总 & 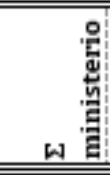 & & 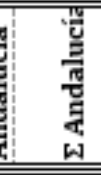 & 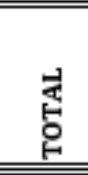 & 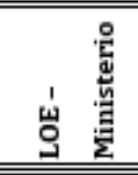 & 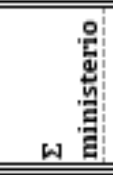 & 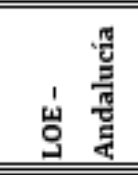 & 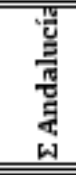 & 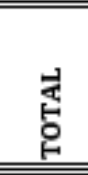 & 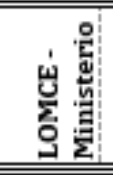 & 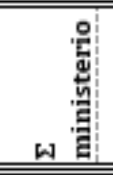 & 岁营 & 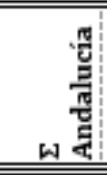 & 렵 \\
\hline \multirow{3}{*}{1} & 11 & 111 & 1 & 0 & 0 & 0 & 0 & 0 & 3 & 3 & 0 & 0 & 3 & 0 & 0 & 0 & 0 & 0 \\
\hline & & 112 & 2 & 0 & 0 & 0 & 0 & 0 & 0 & 0 & 0 & 0 & 0 & 0 & 0 & 0 & 0 & 0 \\
\hline & & 113 & 3 & 0 & 0 & 0 & 0 & 0 & 0 & 0 & 0 & 0 & 0 & 0 & 0 & 0 & 0 & 0 \\
\hline \multirow{12}{*}{2} & 21 & 211 & 1 & 1 & 1 & 1 & 1 & 2 & 3 & 3 & 0 & 0 & 3 & 2 & 2 & 2 & 2 & 4 \\
\hline & & 212 & 2 & 0 & 0 & 0 & 0 & 0 & 0 & 0 & 0 & 0 & 0 & 0 & 0 & 0 & 0 & 0 \\
\hline & & 213 & 3 & 0 & 0 & 0 & 0 & 0 & 1 & 3 & 0 & 0 & 3 & 1 & 3 & 2 & 6 & 9 \\
\hline & 22 & 221 & 1 & 0 & 0 & 0 & 0 & 0 & 0 & 0 & 0 & 0 & 0 & 0 & 0 & 0 & 0 & 0 \\
\hline & 23 & 231 & 1 & 0 & 0 & 0 & 0 & 0 & 0 & 0 & 0 & 0 & 0 & 0 & 0 & 0 & 0 & 0 \\
\hline & & 232 & 2 & 0 & 0 & 0 & 0 & 0 & 0 & 0 & 0 & 0 & 0 & 0 & 0 & 0 & 0 & 0 \\
\hline & & 233 & 3 & 0 & 0 & 0 & 0 & 0 & 0 & 0 & 0 & 0 & 0 & 0 & 0 & 0 & 0 & 0 \\
\hline & 24 & 241 & 1 & 0 & 0 & 0 & 0 & 0 & 0 & 0 & 0 & 0 & 0 & 1 & 1 & 1 & 1 & 2 \\
\hline & 25 & 251 & 1 & 3 & 3 & 2 & 2 & 5 & 1 & 1 & 0 & 0 & 1 & 1 & 1 & 1 & 1 & 2 \\
\hline & & 252 & 2 & 0 & 0 & 0 & 0 & 0 & 0 & 0 & 0 & 0 & 0 & 1 & 2 & 0 & 0 & 2 \\
\hline & & 253 & 3 & 0 & 0 & 1 & 3 & 3 & 1 & 3 & 3 & 9 & 12 & 2 & 6 & 8 & 24 & 30 \\
\hline & & 254 & 3 & 0 & 0 & 0 & 0 & 0 & 1 & 3 & 2 & 6 & 9 & 2 & 6 & 1 & 3 & 9 \\
\hline
\end{tabular}


Tabla 2. Continuación

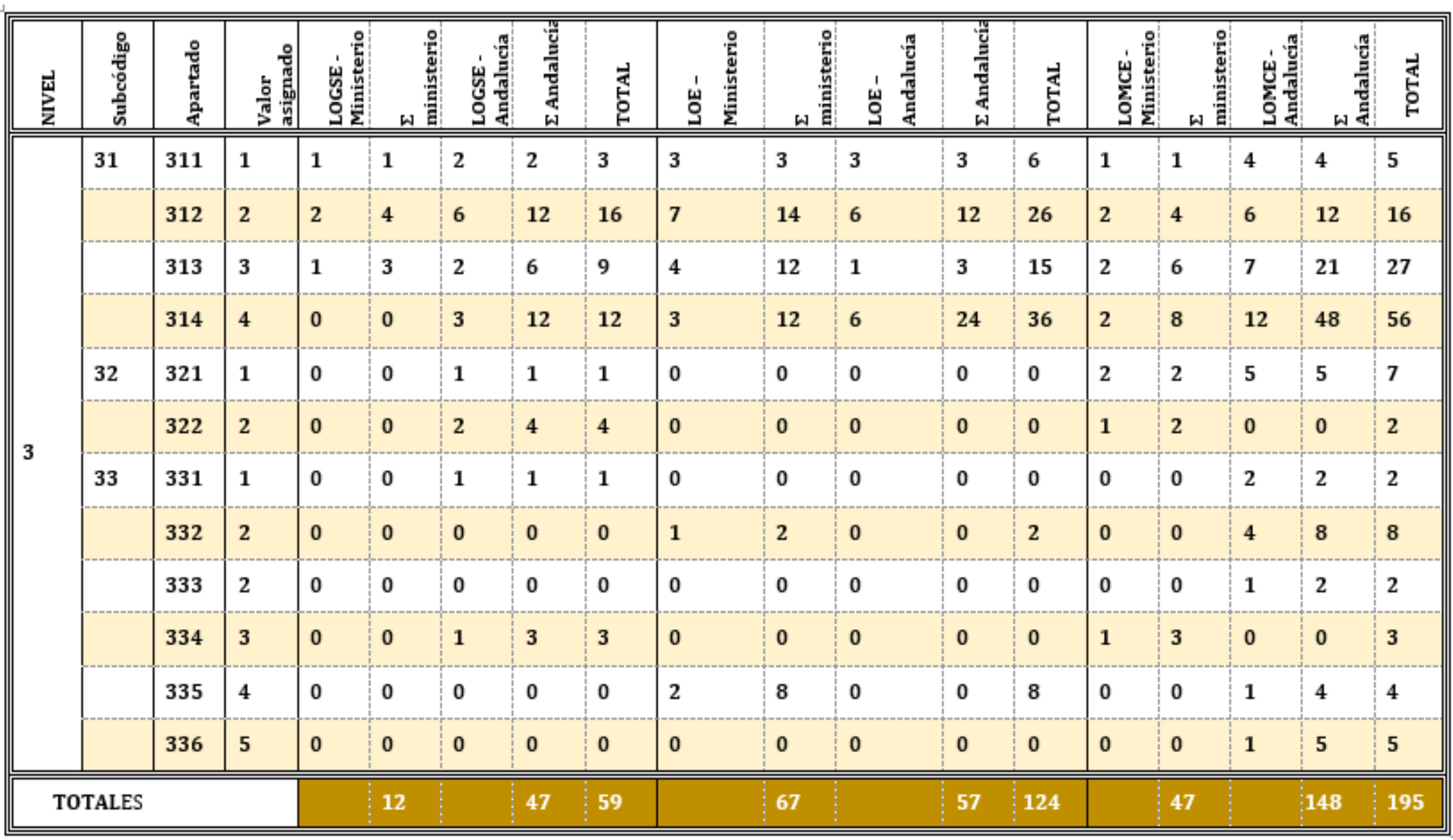


Tabla 3. Matriz - Resumen por categorías principales (Nivel) de la valoración de los textos encontrados

\begin{tabular}{|c|c|c|c|c|c|c|c|c|c|c|c|c|}
\hline \multirow[t]{2}{*}{ Nivel } & \multicolumn{2}{|c|}{ LOGSE Ministerio } & \multicolumn{2}{|c|}{ LOGSE Andalucía } & \multicolumn{2}{|c|}{$\begin{array}{c}\text { LOE } \\
\text { Ministerio }\end{array}$} & \multicolumn{2}{|c|}{$\begin{array}{c}\text { LOE } \\
\text { Andalucía }\end{array}$} & \multicolumn{2}{|c|}{ LOMCE Ministerio } & \multicolumn{2}{|c|}{ LOMCE Andalucía } \\
\hline & 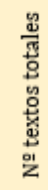 & 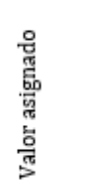 & 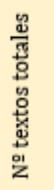 & 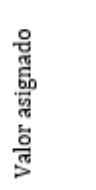 & 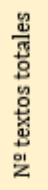 & 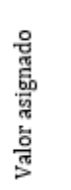 & 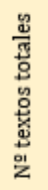 & 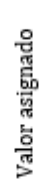 & 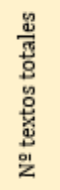 & 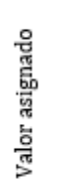 & 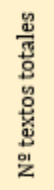 & 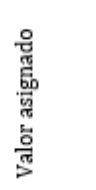 \\
\hline 1 & 0 & 0 & 0 & 0 & 3 & 3 & 0 & 0 & 0 & 0 & 0 & 0 \\
\hline 2 & 4 & 4 & 4 & 6 & 7 & 13 & 5 & 15 & 10 & 21 & 15 & 37 \\
\hline 3 & 4 & 8 & 18 & 41 & 20 & 51 & 16 & 42 & 11 & 26 & 43 & 111 \\
\hline TOTALES & 8 & 12 & 22 & 47 & 30 & 67 & 21 & 57 & 21 & 47 & 58 & 182 \\
\hline
\end{tabular}

\section{Discusión y conclusiones}

El primer aspecto relevante del análisis es la ausencia del propio concepto biodiversidad, que solo se menciona una vez en el ámbito ministerial durante el desarrollo de la LOE, con la denominación diversidad biológica (apartados 112 y 113). Tengamos en cuenta que "en educación, lo que no se nombra no existe" (Pamblanco y Zacarés, 2005).

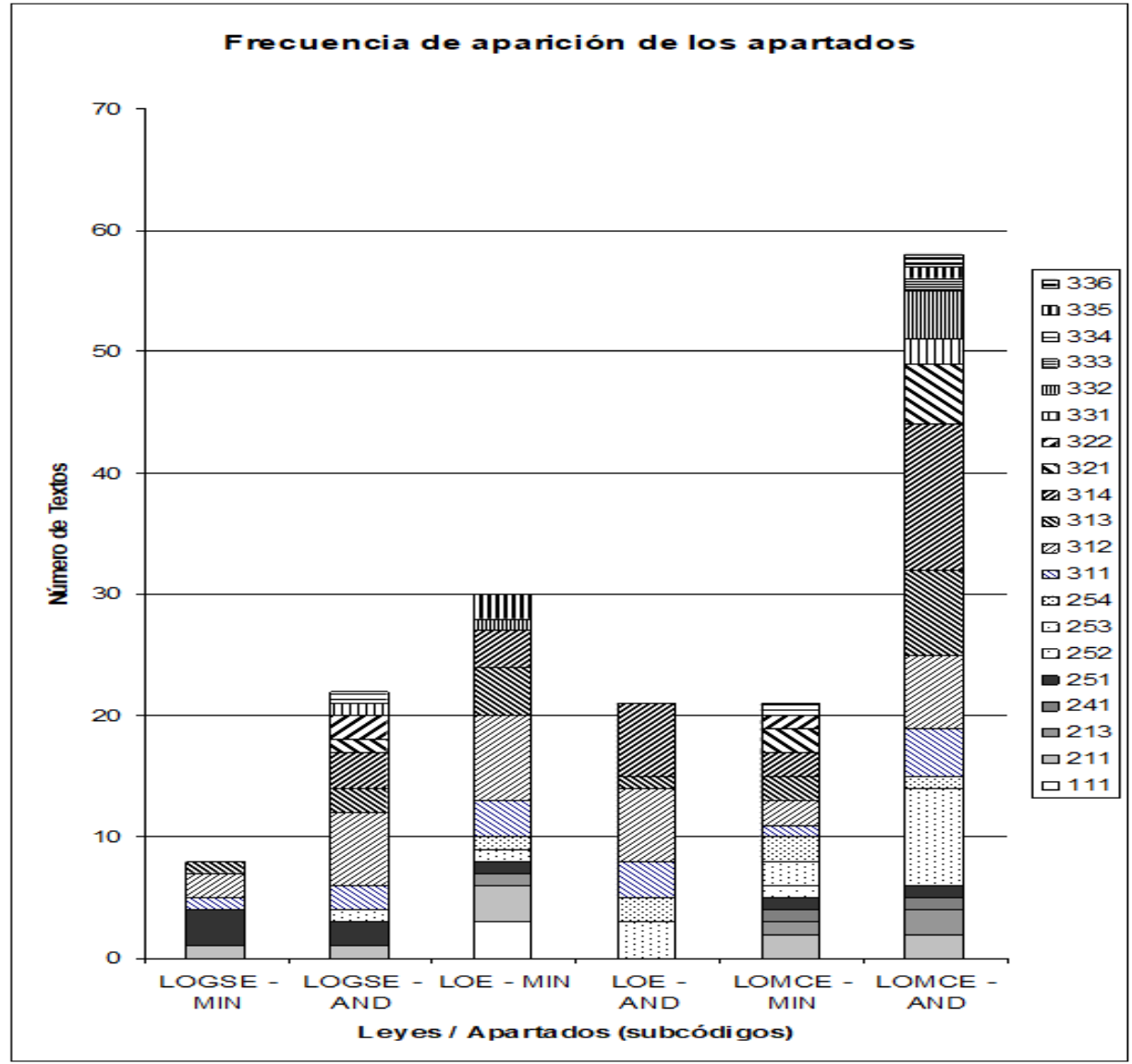

Figura 2. Valoración de los textos encontrados por apartados 
Existe un cierto incremento del tratamiento de la biodiversidad. Pero esta tendencia se rompe con la LOMCE a nivel nacional, donde la biodiversidad pierde tanto en modo extensivo (menos niveles; se habla menos de ella) como en profundidad (menos puntuación; lo que se habla es menos relevante).

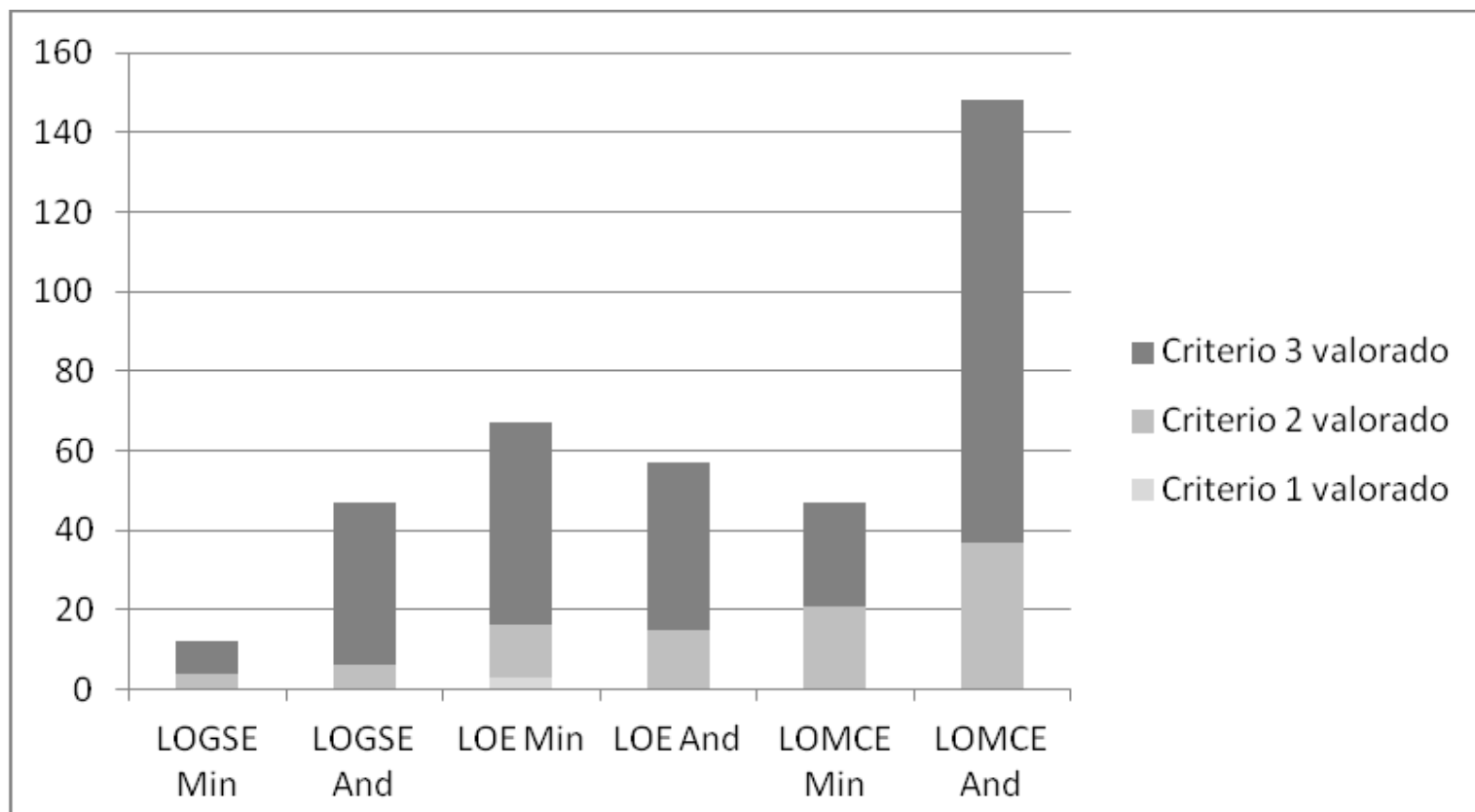

Figura 3. Gráfico de los valores alcanzados evaluados por Nivel

A nivel nacional (no autonómico), se muestra una ausencia casi total de los aspectos más complejos (3.2 y 3.3). Destaca el descenso del tratamiento del Nivel 3 (Nivel Funcional). Esta ausencia transpositiva se traduce en una inadecuada comprensión de los procesos que rigen la biodiversidad y la incidencia del ser humano sobre ellos , y personalmente opinamos que puede entorpecer el desarrollo de posturas comprometidas, justificadas y deliberadamente proactivas para con el medio. La posición antropocéntrica, mayoritaria en todos los currículos educativos, junto al elevado peso natural evolutivo de esta perspectiva en las edades tempranas que nos ocupan (Primaria) consideramos son algunos de los responsables de simplificar excesivamente el metaconcepto de la biodiversidad, soslayando las redes de procesos subyacentes y ponderando en exceso la importancia de consecuencias que nos atañen.

El gráfico de la figura 3 muestra cómo en Andalucía existe una mayor presencia de los aspectos complejos de la biodiversidad. Suponemos esto como algo natural, porque es a nivel autonómico donde se concretan las intervenciones, el aprovechamiento de los recursos específicos que sobre biodiversidad tiene cada uno de los territorios. Sin embargo, con la LOE, el número de citas a nivel autonómico es mucho menor que en el BOE, lo que muestra una incompleta transposición a nivel andaluz.

Tanto a nivel nacional como autonómico existen aspectos que no son tratados en absoluto: 2.2. (Nivel especie) y 2.3. (Nivel genético).

Primeramente, la ausencia del tratamiento de la especie provoca una carencia inicial en biodiversidad. Impide delimitar, distinguir y cuantificar la inmensa variedad de formas, estructuras y funciones dentro del mundo de los distintos seres vivos. Estos contenidos resultan imprescindibles para interpretar correctamente la biodiversidad, 
y la importancia relativa de formas vivas, ambientes y relaciones. Aunque aceptamos que especie es un concepto polémico a nivel especialista, la discusión sobre su contenido epistemológico no tiene por qué volcarse en las aulas, y el alumnado debe ser dotado de una herramienta de clasificación lo suficientemente sólida y duradera como para que: a) resuelva correctamente el problema de distinguir la diversidad de formas vivas atendiendo a criterios funcionales, $y$ no morfológicos; $y$ b) esa herramienta no entre en conflicto con sucesivos aprendizajes sobre el concepto de especie (Blackwell, Powell y Dukes, 2003). En esta aproximación, como nos apunta Bonet (2016), se podría explorar un concepto de especie basado en un criterio funcional (productores primarios, descomponedores, consumidores secundarios, etc.), aprovechando que este tratamiento es suficientemente abundante en los textos escolares.

En segundo lugar, la ausencia del tratamiento del nivel genético podría tener diferentes causas, pero consideramos que la edad del alumnado no debería nunca ser el criterio que lo eliminara.

La pérdida de la biodiversidad y la alteración del medio por impacto de la actividad humana es ya un hecho constatable y cuantificado en múltiples dimensiones de estudio: provocando desplazamientos geográficos y estacionales; alterando la fenología de animales y plantas (Tingley, Monahan, Beissinger y Moritz, 2009), y los nichos de especies especialistas y generalistas (van Heerwaarden y Sgrò, 2014); alterando las estructuras de las cadenas tróficas (Woodward et al., 2012), reduciendo, eliminando o cambiando los recursos disponibles, como los arrecifes de coral o las plataformas de hielo polares (Pratchett, Coker, Jones y Munday 2012), redistribuyendo especies en virtud de la presencia humana (De Angelo, Paviolo y Di Bitetti, 2011); o incrementando la tasa de aparición de especies invasoras (GarcíaDíaz, Ross, Ayres y Cassey, 2015; Strong y Leroux, 2014; Ware et al., 2014 ). Situar la responsabilidad de nuestra especie como agente es imprescindible, evitando lagunas del público en general, pero fundamentalmente en los futuros formadores (VázquezAlonso, y Manassero-Mas, 2015; Martín, Jiménez y Prieto, 2015).

Tal y como postulábamos, consideramos el tratamiento dado a la biodiversidad en las leyes educativas incompleto e incorrecto. A nivel nacional se muestran estas dos carencias, al igual que al nivel autonómico andaluz. El traspaso de competencias en materia de educación a las distintas autonomías no justifica ni explica cómo este tratamiento resulta inadecuado ministerialmente. Existen suficientes niveles de estructuración de la biodiversidad sin transponer como para convertir cualquier desarrollo de la misma en un conocimiento académico antes que una herramienta cognitiva para la toma de decisiones responsables e inaplazables. Un conocimiento que, al menos en los niveles de tratamiento en Primaria, se encuentra sesgado. Estas ausencias son:

1. Tratamiento de la biodiversidad como objeto de conocimiento: este contenido ausente en el currículo provoca en Primaria una confusión de la biodiversidad con la diversidad de especies.

2. Valoración ponderada de la biodiversidad: pérdida del valor de lo escaso, de lo significativo y de lo cognitivamente complejo.

3. Valor propedéutico de la definición de especie: que puede llevar en la Secundaria a problemas en el tratamiento del concepto, hecho ya demostrado 
por otros investigadores (Jiménez-Tejada, Sánchez-Monsalve y GonzálezGarcía, 2013; Jiménez-Tejada et al., 2014).

4. Nivel genético excluido en Primaria: aspecto que redundaría en mejorar la percepción de esta disciplina en Secundaria y Bachillerato. Caurin y MartínezPenella (2013) analizan la importancia de incluir la variabilidad genética en términos idóneos para la transposición de la teoría de la evolución. En el caso de la biodiversidad, el nivel genético posee la misma importancia y debería tener tratamiento semejante.

Valoramos positivamente el incremento paulatino del nivel funcional, más evidente en Andalucía, donde aparece mencionada la práctica totalidad del mismo. Ello incide en una transposición más completa de los aspectos complejos y significativos de la biodiversidad. En el texto de la ley se apuntan, además, formas muy variadas para completar las actuaciones docentes, algo que como docentes de Primaria, también apreciamos.

Consideramos un acierto encarar la biodiversidad en las aulas de Primaria desde su separación curricular en Ciencias Sociales y Ciencias Naturales, aunque ambas sigan desarrollándose dentro de la perspectiva integradora anterior en Primaria, el "Conocimiento del Medio" que entonces designa más un enfoque didáctico que una realidad de conocimiento.

Muchas han sido las propuestas de investigación surgidas tras la revisión de este artículo, principalmente en relación a la estructura interna del Nivel Funcional. Compartimos la opinión del Dr. Bonet de que habría que profundizar en la influencia humana, y un nivel totalmente nuevo, la dimensión "Tendencias de Cambio"; ambas mejorarían la transposición didáctica de la biodiversidad. Porque el correcto tratamiento del nivel funcional, según la aproximación que hemos venido realizando, predispone a solucionar el gran reto educativo en las aulas de Primaria que la pérdida de biodiversidad plantea a nuestro mundo del s. XXI: la toma de posiciones claras y decididas por parte de una sociedad consciente y preparada, que recibe una educación de calidad y comprometida con los valores que el mantenimiento, gestión, protección y recuperación de la biodiversidad exigen. Saber para actuar.

\section{Agradecimientos}

Quisiera, por último, expresar mi agradecimiento a las autoridades que me han acompañado durante el camino de la redacción de este trabajo y a lo redactores anónimos cuyas sugerencias han mejorado el artículo. A los doctores García García y García Abril (Departamento de Didáctica de la UJA), que dieron la idea de la investigación y la guiaron en los inicios, aportando ideas clave en transposición. Al Dr. Hódar Correa por su apoyo. Al Comité de Expertos, doctores Tierno de Figueroa, Pleguezuelos Gómez, Peñas de Giles y Madrona Moreno (compañera que estuvo conmigo hasta el final), y que junto al Dr. Bonet García, realizaron la última de las revisiones de este artículo.

\section{Referencias Bibliográficas}

Armúa de Reyes, A., Guirardo, M. A., y Brataszczuk, E. (2005). Aplicación de metaconceptos en la enseñanza de las Ciencias Naturales. Memorias del Tercer Encuentro de Investigadores en Didáctica de la Biología, Buenos Aires: Argentina.

Bermúdez, G. M. A., De Longhi, A. L., Díaz, S., y Catalán, V. G. (2014). La transposición del concepto de diversidad biológica. Un estudio sobre los libros de texto de la 
educación secundaria española. Enseñanza de las ciencias: revista de investigación y experiencias didácticas, 32(3), 285-302.

Blackmore, L., y Doole, G. J. (2013). Drivers of landholder participation in tender programs for Australian biodiversity conservation. Environmental Science y Policy, 33, 143-153.

Blackwell, W. H., Powell, M. J., y Dukes, G. H. (2003). The problem of student acceptance of evolution. Journal of Biological Education, 37(2), 58-67.

Bosch, M., y Gascón, J. (2006). Twenty-five years of the didactic transposition. ICMI Bulletin, 58, 51-63.

Bosch, M., y Gascón, J. (2009). Aportaciones de la Teoría Antropológica de lo Didáctico a la formación del profesorado de matemáticas de secundaria. En M. J. González, M. T. González, y J. Murillo (Eds.), Investigación en Educación Matemática XIII (pp. 89-113). Santander: SEIEM

Calero Llinares, M., Mayoral García-Berlanga, O., Ull Solís, A. y Vilches Peña, A. (2019). La educación para la sostenibilidad en la formación del profesorado de ciencias experimentales en Secundaria. Enseñanza de las ciencias, 37(1), 157175.

Caurin, C., Martinez-Penella M. J. 2013. Análisis del concepto de biodiversidad en los libros de texto de segundo ciclo de primaria en la Comunidad Valenciana (España). Perfiles educativos, 30(141), 97-114.

Chevallard, I. (1985). La Transposition Didactique. Du savoir savant au savoir enseigné. Le pensée sauvage édition. Grenoble. Cedex. France.

Chevallard, Y. (1992a). Concepts fondamentaux de la didactique: Perspectives apporteés par une approche anthropologique. Recherches en Didactique des Mathématiques, 12(1), 73-112.

Chevallard, Y. (1992b). Fundamental concepts in didactics: Perspectives provided by an anthropological approach. Recherches en Didactique des Mathématiques, special issue, 131-167.

Chevallard, Y. (2002). Organiser l'étude. 3. Écologie y régulation. En J. L. Dorier, M. Artaud, M. Artigue, R. Berthelot y R. Floris (Eds.), Actes de la 11e École d'Été de Didactique des Mathématiques (pp. 41-56). Grenoble, Francia: La Pensée Sauvage.

De Angelo, C., Paviolo, A., y Di Bitetti, M. (2011). Differential impact of landscape transformation on pumas (Puma concolor) and jaguars (Panthera onca) in the Upper Paraná Atlantic Forest. Diversity and Distributions, 17(3), 422-436.

Dangerfield, J. M., y Pik, A. J. (1999). The educational value of an all taxa biodiversity inventory. Journal of Biological Education, 33(2), 76-83.

DeLong, D. C. (1996). Defining biodiversity. Wild!. Soc. Bull . 24, 738-749.

DETR (1999). Sustainable Development Education Panel: First Annual Report 1998. London: HMSO.

Ellis, E. C., y Ramankutty, N. (2008). Putting people in the map: anthropogenic biomes of the world. Frontiers in Ecology and the Environment, 6(8), 439-447. 
Eschenhagen, M. L. (2007). Las cumbres ambientales internacionales y la educación ambiental. Oasis, 12, 39-76.

España Ramos, E., y Prieto Ruz, T. (2010). Problemas socio-científicos y enseñanzaaprendizaje de las ciencias. Investigación en la Escuela, 71, 17-24.

Estrada, F. J. P., y González, G. T. (2004). Aprender investigando, investigar para aprender: el punto de vista de los futuros docentes. Investigación en la Escuela, 54, 5-26.

Fernández-Río, J., y Suárez, C. (2014). Feasibility and students' preliminary views on parkour in a group of primary school children. Physical Education and Sport Pedagogy, 22(1), 281-294.

Fuentes Silveira, M. J., García Barros, S., y Martínez Losada, C. (2008). La presencia de la diversidad en los currículos oficiales. Un estudio comparativo. Actas de los XXIII Encuentros de Didáctica de las Ciencias Experimentales. Almería: Editorial de la Universidad de Almería.

Fuentes Silveira, M. J., García Barros, S., y Martínez Losada, C. (2010). La biodiversidad en el estudio del ecosistema. Un análisis de textos escolares. XXIV Encuentros de Didáctica de las Ciencias Experimentales. Baeza (Jaén): Adapice.

García Baldera, J. M. (2013) ¿Cómo se aborda la Educación Ambiental en los libros de textos de Conocimiento del Medio de Primaria? El caso de la editorial SM. Trabajo Fin de Máster inédito. Universidad de Huelva (España).

García-Díaz, P., Ross, J. V., Ayres, C., y Cassey, P. (2015). Understanding the biological invasion risk posed by the global wildlife trade: propagule pressure drives the introduction and establishment of Nearctic turtles. Global change biology, 21(3), 1078-1091.

Gascón, J. (1993). Desarrollo del conocimiento matemático y análisis didáctico: Del patrón de análisis- síntesis a la génesis del lenguaje algebraico. Recherches en Didactique des Mathématiques, 13(3), 295- 332.

Gascón, J. (2011). Las tres dimensiones fundamentales de un problema didáctico. El caso del álgebra elemental. Revista Latinoamericana de Investigación en Matemática Educativa, 14(2), 203-231.

Gaston, K. J., y Spicer, J. I. (2004). Biodiversity: an introduction. Oxford, United Kingdom: Blackwell Science Ltd.

Gayford, C. (2000). Biodiversity education: A teacher's perspective. Environmental education research, 6(4), 347-361.

Gómez, J. G., y Bernat, F. J. M. (2010). Comó y qué enseñar de la biodiversidad en la alfabetizacion científica. Enseñanza de las ciencias: revista de investigación y experiencias didácticas, 28(2), 175-184.

Herráiz, M. D. C. L., y del Valle López, A. (1995). La Educación Medioambiental en los documentos internacionales: notas para un estudio comparado. Revista complutense de educación, 6(2), 75-94.

Herrería, A. F., y Caveda, J. C. (2010). La ecopedagogía en la formación inicial de maestros. Investigación en la escuela, 71, 39-49. 
Jackson, R. L., Drummond, D. K., y Camara, S. (2007). What is qualitative research?. Qualitative Research Reports in Communication, 8(1), 21-28.

Janghorban, R., Roudsari, R. L., Taghipour, A., y Abbasi, M. (2014). Sexual and Reproductive Rights from Qur'anic Perspective: A Quantitative Content Analysis. Asian Social Science, 11(3), 182-187.

Jiménez Tejada, M. P., Sánchez Monsalve, C., Romero López, M. C., y González García, F. (2014). ¿Cómo se definen los conceptos de población y especie en los libros de conocimiento del medio de primaria? Actas de los 26 Encuentros de Didáctica de las Ciencias Experimentales. (pp. 866-873). Huelva, 10-12 de septiembre de 2014.

Jiménez-Tejada, M. P., Sánchez-Monsalve, C., y González-García, F. (2013). How Spanish primary school students interpret the concepts of population and species. Journal of Biological Education, 47(4), 232-239.

Libarkin, J. C., and J. P. Kurdziel. 2002. Research Methodologies in Science Education: The Qualitative-Quantitative Debate. Journal of Geoscience Education 50 (1): 78-86.

Lincoln, Y. S., y Guba, E. G. (1985). Naturalistic Inquiry. Newbury Park, C. A.: Sage.

Lindemann-Matthies, P., Constantinou, C., Junge, X., Köhler, K., Mayer, J., Nagel, U., y Kadji - Beltran, C. (2009). The integration of biodiversity education in the initial education of primary school teachers: four comparative case studies from Europe. Environmental Education Research, 15(1), 17-37.

Makarova, E., y Birman, D. (2015). Cultural transition and academic achievement of students from ethnic minority backgrounds: a content analysis of empirical research on acculturation. Educational Research, 57(3), 305-330.

Martín Gámez, C., Jiménez López, M. A., y Prieto Ruz, T. (2015). Tendencias del profesorado de ciencias en formación inicial sobre las estrategias metodológicas en la enseñanza de las ciencias. Estudio de un caso en Málaga. Enseñanza de las Ciencias, 33(1), 167-184.

Martín-Vivaldi Martínez, E. M., Gutiérrez Pérez, J. y Arjona Díaz, S. A. (2000). Buena marcha de la red andaluza de ecoescuelas. Aula verde, 21, 10.

Noss, R. F. (1990). Indicators for monitoring biodiversity: a hierarchical approach. Conservation biology, 355-364.

Noss, R. F., y Cooperrider, A. (1994). Saving nature's legacy: protecting and restoring biodiversity. Washington, D.C: Island Press

Núñez, I., González-Gaudiano, É., y Barahona, A. (2003). La Biodiversidad: Historia y contexto de un concepto. Interciencia, 28(7), 387-393.

Pamblanco, A. Z., y Zacarés, A. (2005). La violencia de género explicada a mi hijo. Carena Editors, Sl.

Peña, A. V., Gil, D., y Cañal, P. (2010). Educación para la sostenibilidad y educación ambiental. Investigación en la Escuela, 71, 5-15. 
Pratchett, M. S., Coker, D. J., Jones, G. P., y Munday, P. L. (2012). Specialization in habitat use by coral reef damselfishes and their susceptibility to habitat loss. Ecology and evolution, 2(9), 2168-2180.

PNUMA (2002). Estado del medio ambiente y medidas normativas: 1972-2002. Biodiversidad Madrid; Grupo Mundi-Prensa Libros.

Redford, K. H., y Richter, B. D. (1999). Conservation of Biodiversity in a World of Use. Conservation Biology, 13(6) 1246-1256. doi: 10.1046/j.15231739.1999.97463.

Saldaña, J. (2009). The Coding Manual for Qualitative Researchers. London: SAGE.

Sánchez-Cañete, F. J. S., y Pedrajas, A. P. (2010). La comprensión de conceptos de ecología y sus implicaciones para la educación ambiental. Revista Eureka sobre enseñanza y divulgación de las ciencias, 7(número extraordinario), 271-285.

Schnurr, M. A., De Santo, E. M., Green, A. D., y Taylor, A. (2015). Investigating Student Perceptions of Knowledge Acquisition within a Role-Play Simulation of the Convention on Biological Diversity. Journal of Geography, 114(3), 94-107.

Strong, J. S., y Leroux, S. J. (2014). Impact of non-native terrestrial mammals on the structure of the terrestrial mammal food web of Newfoundland, Canada. Plos one, 9(8), e106264. https://doi.org/10.1371/journal.pone.0106264.

Swingland, I. R. (2001). Biodiversity, definition of. En S. A. Levin, (editor-in-chief), Encyclopedia of Biodiversity, Vol. I (pp. 377- 391) San Diego, California: Academic Press.

Tellería, J. L. (2012). Introducción a la conservación de las especies. Valencia: Tundra Ediciones.

Tingley, M. W., Monahan, W. B., Beissinger, S. R., y Moritz, C. (2009). Birds track their Grinnellian niche through a century of climate change. Proceedings of the National Academy of Sciences, 106 (Supplement 2), 19637-19643.

Valverde, G. A. (2009). Estándares y evaluación. En S. Schwartzman y C. Cox (Eds.), Políticas Educativas y Cohesión Social en América Latina, (pp. 57-88). Santiago, Chile: Colección Cieplan.

Van Heerwaarden, B., y Sgrò, C. M. (2014). Is adaptation to climate change really constrained in niche specialists? Proceedings of the Royal Society of London B: Biological Sciences, 281, 20140396. http://dx.doi.org/10.1098/rspb.2014.0396.

Vázquez-Alonso, A., y Manassero-Mas, M. A. (2015). Towards a development of initial science teacher training based on research. Revista Española de Pedagogía, 73(261), 343-363.

Vázquez-Ben, L. y Bugallo-Rodríguez, A. (2018). El modelo de evolución biológica en el curriculum de Educación Primaria: un análisis comparativo en distintos países. Revista Eureka sobre Enseñanza y Divulgación de las Ciencias, 15(3), 3101.

Ware, C., Berge, J., Sundet, J. H., Kirkpatrick, J. B., Coutts, A. D., Jelmert, A., ..., y Alsos, I. G. (2014). Climate change, non-indigenous species and shipping: assessing the 
risk of species introduction to a high-Arctic archipelago. Diversity and Distributions, 20(1), 10-19.

Wilson, E. O. (1986). Biodiversity (Papers from the 1st National Forum on Biodiversity, September 1986, Washington, D.C.). Paperback, October 6, 1996. Harvard: National Academy of Sciences/Smithsonian Institution.

Wilson, E. O. (1988). The current state of biological diversity. En E. O. Wilson (Ed.) y F. M. Peter (Associate Ed.), Biodiversity, (pp. 3-18). Washington D. C.: National Academy Press.

Woodward, G., Brown, L. E., Edwards, F. K., Hudson, L. N., Milner, A. M., Reuman, D. C., y Ledger, M. E. (2012). Climate change impacts in multispecies systems: drought alters food web size structure in a field experiment. Philosophical Transactions of the Royal Society of London B: Biological Sciences, 367, 29902997. 